\title{
Editorial
}

\section{Intervenções não farmacológicas para o enfrentamento à epidemia da COVID-19 no Brasil}

doi: 10.5123/51679-49742020000200009

\author{
Nonpharmaceutical interventions for tackling the COVID-19 epidemic in Brazil \\ Intervenciones no farmacéuticas para abordar la epidemia de COVID-19 en Brasil
}

\begin{abstract}
A CoVID-19 foi detectada em Wuhan, China, em dezembro de 2019. Com o crescimento no número de casos, óbitos e países afetados, a Organização Mundial da Saúde (OMS) declarou que o evento constituía uma Emergência de Saúde Pública de Importância Internacional (ESPII), em 30 de janeiro de 2020. ${ }^{1}$ No Brasil, a epidemia foi declarada Emergência em Saúde Pública de Importância Nacional (ESPIN), em 3 de fevereiro de 2020. ${ }^{2,3}$ Com a notificação de mais de 110 mil casos e 4 mil óbitos em países de todos os continentes, a OMS declarou a pandemia de COVID-19, em 11 de março de 2020.

A elevada infectividade do SARS-CoV-2, agente etiológico da COVID-19, na ausência de imunidade prévia na população humana, bem como de vacina contra este vírus, faz com que o crescimento do número de casos seja exponencial. Nesse contexto, são indicadas intervenções não farmacológicas (INF), visando inibir a transmissão entre humanos, desacelerar o espalhamento da doença, e consequentemente diminuir e postergar o pico de ocorrência na curva epidêmica. ${ }^{4}$ Com isso, é possível reduzir a demanda instantânea por cuidados de saúde e mitigar as consequências da doença sobre a saúde das populações, incluindo a minimização da morbidade e da mortalidade associadas (Figura 1$){ }^{5}$
\end{abstract}

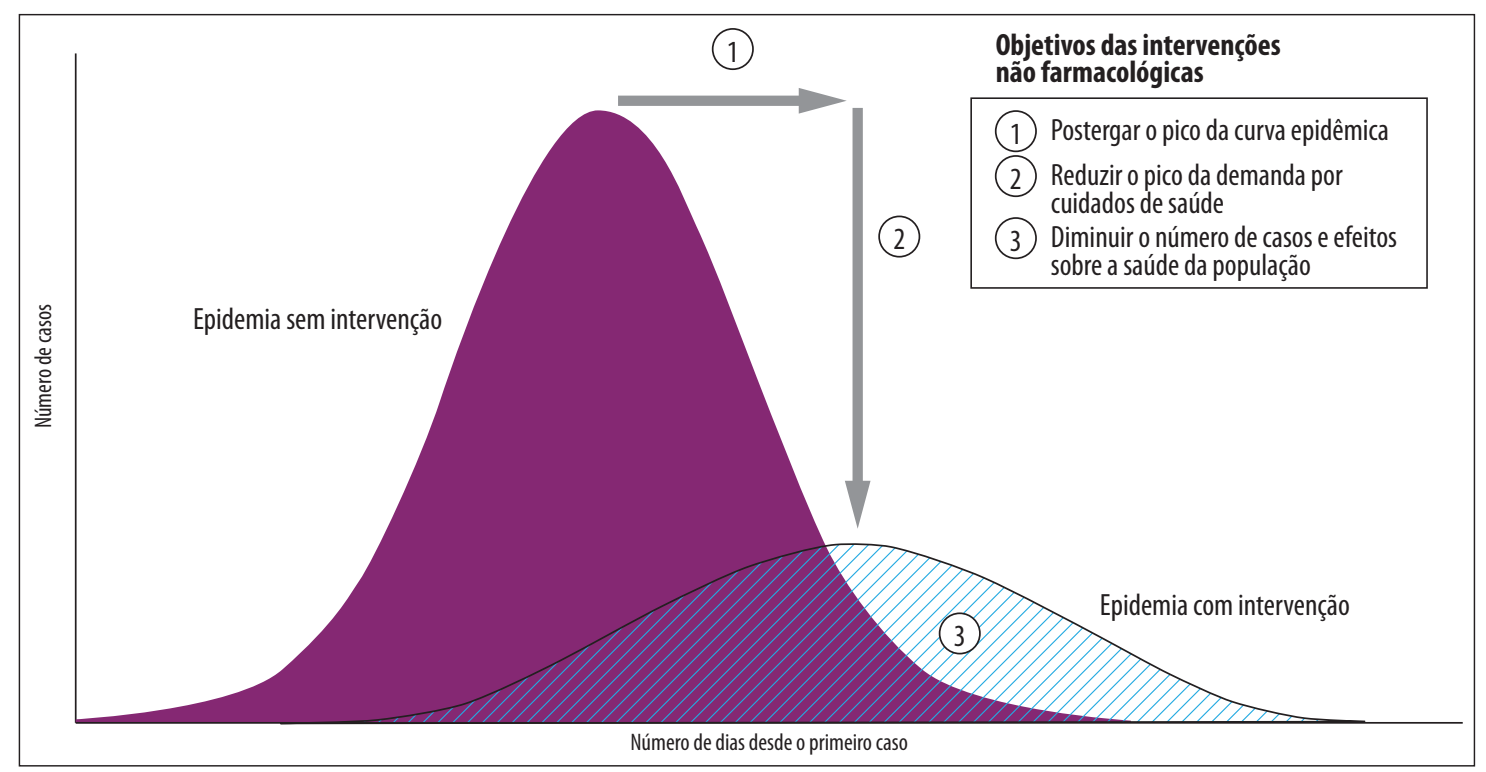

Figura 1 - Curva epidêmica hipotética mostrando o curso normal da epidemia e 0 achatamento da curva esperado com a adoção de intervenções não farmacológicas

Fonte: Adaptado de Centers for Disease Control and Prevention (CDC), 2007. 5,6 
As INF são medidas de saúde pública com alcance individual, ambiental e comunitário. As medidas individuais incluem a lavagem das mãos, a etiqueta respiratória e o distanciamento social. 0 distanciamento social, por sua vez, abrange 0 isolamento de casos, a quarentena aplicada a contatos, e a prática voluntária de não frequentar locais com aglomerações de pessoas. ${ }^{5}$

Outra medida individual é o uso de máscaras, recomendado para indivíduos com infecção pelo coronavírus confirmada ou suspeita, e seus cuidadores. A OMS recomenda que pessoas assintomáticas não usem máscaras, por falta de evidência de sua efetividade para redução da transmissão da influenza, além de dar a falsa sensação de proteção. Contudo, não existem estudos sobre a efetividade do uso de máscaras por pessoas assintomáticas para prevenção da transmissão da COVID-19.

As medidas ambientais referem-se ao arejamento e exposição solar de ambientes, e à limpeza rotineira de ambientes e superfícies, procedimentos que ajudam a eliminar os vírus. ${ }^{5} 0$ SARS-CoV-2, assim como 0 vírus influenza, pode permanecer estável fora do corpo humano, em aerossóis e diferentes superfícies, por até três dias, como no caso do plástico e do aço inoxidável. ${ }^{7}$ Deve-se ter atenção especial com a limpeza de botões de elevador, corrimãos, apoios em veículos de transporte público, maçanetas de portas, teclados de máquinas de pagamento com cartão, smartphones, estações de trabalho, entre outros objetos e superfícies que, ao estarem contaminados, podem contribuir para a propagação dos vírus.

As medidas comunitárias são ações tomadas por gestores, empregadores e/ou líderes comunitários para proteger a população. Incluem a restrição ao funcionamento de escolas, universidades, locais de convívio comunitário, transporte público, além de outros locais onde há aglomeração de pessoas, como eventos sociais, esportivos, teatros, cinemas e estabelecimentos comerciais, que não são caracterizados como prestadores de serviços essenciais. ${ }^{5}$

0 momento do início e a duração das diversas INF comunitárias influenciará seu impacto. É um grande desafio determinar o melhor momento para o início de tais intervenções, uma vez que sua implementação precoce pode resultar em dificuldades econômicas e sociais sem benefício à saúde pública e, com passar do tempo, resultar em "fadiga da intervenção" e perda da adesão por parte da população. Por outro lado, a implementação após a disseminação extensiva da doença pode limitar os benefícios para a saúde pública. Precisa ocorrer cedo 0 suficiente para impedir a subida íngreme inicial no número de casos, e ser longa o suficiente para cobrir o pico da curva epidêmica prevista. ${ }^{5}$

Diante das lacunas de conhecimento inerentes a uma doença nova e considerando-se a similaridade entre os padrões de comportamento do SARS-CoV-2 e dos vírus causadores da influenza pandêmica, as estratégias adotadas nos planos de contingência para influenza pandêmica também estão sendo consideradas para a pandemia da COVID-19. A orientação do Centro de Controle e Prevenção de Doenças (Centers for Disease Control and Prevention - CDC), dos Estados Unidos, sobre a mitigação da influenza pandêmica, aponta que o momento de início das intervenções deve ser baseado em avaliações da severidade da doença. ${ }^{8}$ Avaliação da COVID-19, conforme o Quadro de Avaliação da Gravidade Pandêmica (Pandemic Severity Assessment Framework - PSAF), do CDC, apontou que a doença apresenta elevada transmissibilidade e gravidade clínica. ${ }^{9}$ Face à gravidade da doença, e à intensidade da transmissão comunitária, é justificado o uso de INF comunitárias para mitigação da pandemia da COVID-19. ${ }^{10}$

Medidas como quarentena em domicílio para pessoas infectadas, distanciamento social e redução de reuniões públicas, como reuniões em igrejas e fechamento de escolas, foram implementadas durante a pandemia de influenza de 1918 a 1919, em várias cidades dos Estados Unidos. ${ }^{11}$ Na pandemia de influenza de 2009, verificou-se que a quarentena doméstica foi factível e alcançou boa adesão na Austrália. ${ }^{12} \mathrm{~A}$ experiência da China sugere que as INF, que incluíram medidas rigorosas de bloqueio da circulação de pessoas, como aquelas adotadas na cidade de Wuhan a partir de 23 de janeiro de 2020, contribuíram para a supressão da epidemia da COVID-19 naquele país., ${ }^{43}$

No Brasil, em 6 de fevereiro de 2020, foi sancionada a Lei $\mathrm{n}^{0}$ 13.979, que dispõe sobre as medidas para enfrentamento da epidemia da COVID-19 e elenca as INF comunitárias que podem ser adotadas. As Unidades da Federação (UFs) passaram a adotar tais medidas a partir da segunda semana de março de 2020. São exemplos 0 estado do Rio de Janeiro (Decreto $\mathrm{n}^{0} 46.970$, de 13 de março de 2020), ${ }^{14} 0$ Distrito Federal (Decreto $\mathrm{n}^{0} 40.520$, de 14 de março de 2020), ${ }^{15} 0$ município de São Paulo (Decreto $\mathrm{n}^{0} 59.283$, de 16 de março de 2020 ${ }^{16}$ e 0 estado de Santa Catarina (Decreto $\mathrm{n}^{0} 515$, de 17 de março de 2020) ${ }^{17}$ que, posteriormente, aprovaram novos decretos 
estabelecendo medidas mais rigorosas. É esperado e desejável que as ações de enfrentamento sejam revistas e alteradas à medida que a epidemia evolui. ${ }^{5}$ Destaca-se o estado de São Paulo, o mais populoso do país, que adotou medidas rigorosas de quarentena a partir de 24 de março de $2020 .^{18}$

Cumpre destacar que, para a implementação de determinadas INF, devem ser consideradas condições particulares de vulnerabilidade de subgrupos populacionais, como moradores de rua, população carcerária, idosos institucionalizados ${ }^{18}$ residentes em domicílios onde há aglomeração de pessoas, não há ventilação adequada, ou não existe água canalizada, migrantes, portadores de necessidades especiais, pessoas que vivem sozinhas, entre outros.

Ademais, a adoção de tais medidas tem impactos importantes nas atividades diárias, nas vidas das pessoas e na sociedade. Por exemplo, crianças deixam de ir às escolas, tendo seus estudos interrompidos, e perdem 0 acesso à alimentação escolar. A restrição do contato social pode trazer consequências para a saúde mental e física das pessoas, particularmente das crianças e dos idosos. ${ }^{4,19}$ Trabalhadores podem ser impedidos de se dirigir ao local de trabalho, necessitar alterar suas rotinas para a realização de trabalho remoto, ou mesmo perder seus empregos ou fontes de renda. Particularmente as mulheres e crianças ficam mais vulneráveis à violência doméstica e intrafamiliar. Também são esperadas perdas econômicas para pessoas, famílias, empresas e países.

Nesse contexto, é fundamental a atuação do Sistema Único de Saúde (SUS) e das demais áreas do sistema de proteção social de forma articulada, de modo a se favorecer a adesão das pessoas às INF e minimizar os impactos deletérios das medidas comunitárias. A proteção da saúde pública deverá ser norteadora das decisões a serem tomadas pelos gestores. É fundamental que essas decisões sejam baseadas nas melhores evidências disponíveis e comunicadas de forma transparente, para se promover a confiança da população. As orientações das autoridades e a adesão das pessoas às INF serão determinantes para o curso da epidemia da COVID-19 no Brasil.

Leila Posenato Garcia' - (1) orcid.org/0000-0003-1146-2641

Elisete Duarte ${ }^{2}$ - (1) orcid.org/0000-0002-0501-0190

'Instituto de Pesquisa Econômica Aplicada, Diretoria de Estudos e Políticas Sociais, Brasília, DF, Brasil

${ }^{2}$ Secretaria de Vigilância em Saúde do Ministério da Saúde, Coordenação-Geral de Desenvolvimento da Epidemiologia em Serviço, Brasília, DF, Brasil

\section{Referências}

1. World Health Organization. Statement on the second meeting of the International Health Regulations (2005) Emergency Committee regarding the outbreak of novel coronavirus (2019-nCoV) [Internet]. Geneva: World Health Organization; 2020 [cited 2020 Mar 27]. Available from: https:/www.who.int/news-room/detail/30-01-2020-statement-on-the-second-meetingof-the-international-health-regulations-(2005)-emergency-committee-regarding-the-outbreak-of-novel-coronavirus-(2019ncov)

2. Ministério da Saúde (BR). Portaria MS/GM n. 188, de 3 de fevereriro de 2020. Declara Emergência em Saúde Pública de importância Nacional (ESPIN) em decorrência da Infecção Humana pelo novo Coronavírus (2019-nCoV) [Internet]. Diário Oficial da União, Brasilia (DF), 2020 fev 4 [citado 2020 mar 27]; Seção 1:1. Disponível em: http://www.in.gov.br/web/dou/-/ portaria-n-188-de-3-de-fevereiro-de-2020-241408388

3. Croda JHR, Garcia LP. Resposta imediata da Vigilância em Saúde à epidemia da COVID-19. Epidemiol Serv Saúde [Internet]. 2020 [citado 2020 Mar 26];29(1):e2020002. Disponível em: https://doi.org/10.5123/s1679-49742020000100021

4. Anderson RM, Heesterbeek H, Hollingsworth TD. How will country-based mitigation measures influence the course of the COVID-19 epidemic? Lancet [Internet]. 2020 Mar [cited 2020 Mar 27];395(10228):931-4. Available from: https:/doi. org/10.1016/S0140-6736(20)30567-5

5. Qualls N, Levitt A, Kanade N, Wright-Jegede N, Dopson S, Biggerstaff M, et al. Community mitigation guidelines to prevent pandemic influenza — United States, 2017MMWR Recomm Rep [Internet]. 2017 Apr [cited 2020 Mar 27];66(1):1-32. Available from: https://doi.org/10.15585/mmwr.rr6601a1

6. Source: Adapted from: CDC. Interim pre-pandemic planning guidance: community strategy for pandemic influenza mitigation in the United States — early, targeted, layered use of nonpharmaceutical interventions. Atlanta, GA: US Department of Health and Human Services, CDC; 2007. https://stacks.cdc.gov/view/cdc/11425. 
7. van Doremalen N, Bushmaker T, Morris DH, Holbrook MG, Gamble A, Williamson BN, et al. Aerosol and Surface Stability of SARS-CoV-2 as Compared with SARS-CoV-1. N Engl J Med [Internet]. 2020 Mar [cited 2020 Mar 27]. Available from: https:// www.nejm.org/doi/full/10.1056/NEJMc2004973

8. Center for Disease Control and Prevention (USA). Departamento f Health \& Human Services. Interim pre-pandemic planning guidance: community strategy for pandemic influenza mitigation in the United States: early, targeted, layered use of nonpharmaceutical interventions [Internet]. [Washington, D.C.]: Center for Disease Control and Prevention; 2007 [cited 2020 Mar 27]. 108 p. Available from: https:/www.cdc.gov/flu/pandemic-resources/pdf/community_mitigation-sm.pdf

9. Freitas ARR, Napimoga M, Donalisio MR. Análise da gravidade da pandemia de COVID-19. Epidmiol Serv Saúde. No prelo 2020.

10. Center for Disease Control and Prevention (USA). Implementation of mitigation strategies for communitieswith local COVID-19 transmission [Internet]. [Washington, D.C.]: Center for Disease Control and Prevention; 2019 [cited 2020 Mar 27]. 10 p. Available from: https:/www.cdc.gov/coronavirus/2019-ncov/downloads/community-mitigation-strategy.pdf

11. Markel H, Stern AM, Navarro JA, Michalsen JR, Monto AS, Di Giovanni C. Nonpharmaceutical influenza mitigation strategies, US communities, 1918-1920 pandemic. Emerg Infect Dis [Internet]. 2006 Dec [cited 2020 Mar 27];12(12):1961-4. Available from: https://doi.org/10.3201/eid1212.060506

12. 12 Teh B, Olsen K, Black J, Cheng AC, Aboltins C, Bull K, et al. Impact of swine influenza and quarantine measures on patients and households during the H1N1/09 pandemic. Scand J Infect Dis [Internet]. $2012 \mathrm{Apr}$ [cited 2020 Mar 27];44(4):289-96. Available from: https://doi.org/10.3109/00365548.2011.631572

13. Kraemer MU, Yang CH, Gutierrez B, Wu CH, Klein B, Pigott DM, et al. The effect of human mobility and control measures on the COVID-19 epidemic in China. Science [Internet]. 2020 Mar [cited 2020 Mar 27]:eabb4218. Available from: https:// science.sciencemag.org/content/early/2020/03/25/science.abb4218

14. Governo do Estado do Rio de Janeiro. Secretaria de Estado da Casa Civil e Governança. Decreto n. 46.970, de 13 de março de 2020. Dispõe sobre medidas temporárias de prevenção ao contágio e de enfrentamento da propagação decorrente do novo coronavírus (COVID-19), do regime de trabalho de servidor público e contratado, e dá outras providências [Internet]. Diário Oficial do Estado do Rio de Janeiro, Rio de Janeiro (RJ), 2020 mar 13 [citado 2020 mar 27]. Disponível em: http:/www.fazenda.ri.gov.br/sefaz/content/conn/UCMServer/path/Contribution\%20Folders/site_fazenda/Subportais/ PortalGestaoPessoas/Legislações\%20SILEP/Legislações/2020/Decretos/DECRET0\%20N\%2046.970\%20DE\%2013\%20DE\%20 MARÇ0\%20DE\%202020_MEDIDAS\%20TEMPORÁRIAS\%20PREVENÇÃ0\%20COR0NAVÍRUS.pdf?lve

15. Governo do Distrito Federal (BR). Decreto n. 40.550, de 23 de março de 2020. Dispõe sobre as medidas para enfrentamento da emergência de saúde pública de importância internacional decorrente do novo coronavírus, e dá outras providências [Internet]. Diário Oficial do Distrito Federal, Brasília (DF), 2020 mar 23 [citado 2020 mar 27]; Edição Extra, Disponível em: http://www.sinj.df.gov.br/sinj/Norma/ed3d931f353d4503bd35b9b34fe747f2/Decreto_40520_14_03_2020.html

16. Prefeitura de São Paulo (SP). Casa Civil. Decreto n. 59.283, de 16 de março de 2020. Declara situação de emergência no Município de São Paulo e define outras medidas para o enfrentamento da pandemia decorrente do coronavírus [Internet]. São Paulo: Prefeitura; 2020 [citado 2020 mar 27]. Disponível em: https:/leismunicipais.com.br/a/sp/s/sao-paulo/ decreto/2020/5929/59283/decreto-n-59283-2020-declara-situacao-de-emergencia-no-municipio-de-sao-paulo-e-defineoutras-medidas-para-o-enfrentamento-da-pandemia-decorrente-do-coronavirus

17. Governo do Estado de Santa Catarina. Decreto n. 515, de 17 de março de 2020. Declara situação de emergência em todo o território catarinense, nos termos do COBRADE n ${ }^{0}$ 1.5.1.1.0 - doenças infecciosasvirais, para fins de prevenção e enfrentamento à COVID-19, e estabelece outras providências [Internet]. Florianópolis: Governo do Estado de Santa Catarina; 2020 [citado 2020 mar 27]. Disponível em: https:/www.sc.gov.br/images/Secom_Noticias/Documentos/NERS\%C3\%830_ ASSINADA.pdf

18. Governo do Estado de São Paulo. Decreto n. 64.881, de 22 de março de 2020. Decreta quarentena no Estado de São Paulo, no contexto da pandemia do COVID-19 (Novo Coronavírus), e dá providências complementares [Internet]. São Paulo: Governo do Estado de São Paulo; 2020 [citado 2020 mar 27]. Disponível em: https:/www.saopaulo.sp.gov.br/wp-content/ uploads/2020/03/decreto-quarentena.pdf

19. Armitage R, Nellums LB. COVID-19 and the consequences of isolating the elderly. Lancet Public Health [Internet]. 2020 Mar [cited 2020 Mar 27];pii:S2468-2667(20)30061-X. Available from: https:/doi.org/10.1016/S2468-2667(20)30061-X 